\title{
Estudio de las Prácticas Culturales en sus Relaciones con Agricultura, Suelo y Ambiente
}

\author{
Elsa P. Sampaio \\ Universidade de Évora, Departamento de Geociencias, Colégio Luís António Verney, Rua Romão \\ Ramalho-59, 7000-671 Évora-Portugal (e-mail: ems@uevora.pt)
}

\begin{abstract}
Resumen
Para evaluar los efectos a largo plazo de diferentes prácticas culturales en el medioambiente y suelo, se realizó un estudio comparativo entre un sistema de producción de cereales de secano de conservación y otro tradicional, a lo largo de 10 años consecutivos. El sistema de conservación ha recurrido a siembras directas, mientras que el tradicional al laboreo del suelo. En un suelo tipo Vertic Cambisol en Portugal se evaluó el contenido en materia orgánica, la relación carbono/nitrógeno, la densidad aparente, la cantidad, dimensión y estabilidad de la agregación, la macroporosidad de campo y la capacidad de almacenamiento de agua. Los resultados han demostrado que en el sistema de conservación hay una variación en las características del suelo que conducen a una mayor capacidad para producir vegetales, gestionar los recursos hídricos, secuestrar carbono orgánico y resistir a la erosión. Estos resultados tienen implicaciones positivas en la sustentabilidad de los sistemas agrícolas que recurren a siembras directas en suelos de textura fina de las áreas mediterráneas continentales en Europa.
\end{abstract}

Palabras clave: suelo, siembra directa, prácticas culturales, erosión, almacenamiento de agua

\section{Cultural Practices Study in its Relationships with Agriculture, Soil and Environment}

\begin{abstract}
In order to evaluate the long-term effects of different cultural practices on the environment and in the soil, a comparative study between a dryland conservation cropping system of cereals and a more traditional one, along 10 consecutive years, was done. In the conservation system direct seeding has been used, while in the traditional one soil tillage was employed. On a soil of the type Vertic Cambisol of Portugal, the following parameters were evaluated: content in organic matter, carbon/nitrogen ratio, bulk density, quantity, size and aggregation stability, field macroporosity and capacity of water storage. The comparative results have showed that in the conservative system there is a change in soil characteristics that drive to an enhanced vegetal production capacity, water resources management, organic carbon sequestration and erosion resistance. These results have positive implications on the sustainability of the agricultural systems that employ direct seeding in soils of clay texture of the Mediterranean continental areas in Europe.
\end{abstract}

Keywords: soil, direct seeding, cultural practices, erosion, water storage 


\section{INTRODUCCIÓN}

Los procesos de degradación del suelo, tales como; la desertificación, la erosión, la reducción del contenido en materia orgánica, la contaminación, la salinización, la compactación y la reducción de la biodiversidad, pueden causar la degradación de algunas de las características que confieren al suelo la capacidad para ejercer sus funciones. Sin embargo, la agricultura también puede aportar soluciones para muchos de los desafíos actuales, tales como el cambio climático y otras perturbaciones ambientales. Autores como, Chatskikh y Olesen (2007); Xiao-Bin et al. (2006) y Holland (2004) han llamado la atención para muchas cuestiones relacionadas con la llamada agricultura ecológica.

Por lo tanto, uno de los objetivos actuales de la agricultura es alcanzar el verdadero equilibrio entre una producción competitiva y el respeto por el medio ambiente. Es por eso, antes que la mayoría de los suelos explotados por el hombre pierdan su riqueza, que es necesario un cambio en los hábitos. El agricultor debe modificar sus sistemas de explotación de la tierra en base a las potencialidades edafoclimáticas y así alcanzar el referido equilibrio, es decir, métodos de cultivo sustentables. Según Bastin et al. (2002), los sistemas de producción que se preocupan por la protección y conservación de recursos como el agua, el suelo y la materia orgánica entre otros, parecen ser los más favorables para el desarrollo de hábitat funcionales. Además Xiao-Bin et al. (2006), valoran positivamente la relación entre los sistemas de conservación y el uso sustentable a lo que afirmen que existen diversos potenciales beneficios en los sistemas de conservación, tales como en el secuestro de carbono, disponibilidad de nutrientes y productividad.

Carvalho (2002), afirma que la actividad agrícola y la protección del suelo o la economía y el medio ambiente no son objetivos en conflicto sino dos caras de la misma moneda. Además, según este autor, existe una clara responsabilidad del hombre en cuanto al estado de degradación actual de la mayoría de los suelos portugueses. Carvalho et al. (2002), observaron que, por ejemplo, el bajo contenido en materia orgánica de los suelos portugueses no es una consecuencia inevitable del clima mediterráneo, sino el resultado de sistemas de movilización demasiado intensivos y de sistemas de cultivos que dejan poco o ningún residuo orgánico en el suelo.

Con este fin, en las últimas décadas, se han llevado a cabo, en distintos países del mundo, gran número de investigaciones técnicas y científicas abarcando gran diversidad de suelos, con distintas características y cultivos (Elder y Rattan, 2008; Moreno et al., 2008; Castro et al., 2008; Hermle et al., 2008; Chatskikh y Olesen, 2007; Abrisqueta et al., 2007; Govaerts et al., 2007; Jin et al., 2007; Reynolds et al., 2007; Pereira et al., 2007; Xiao-Gang et al., 2007; Lal et al., 2007; Govaerts et al., 2006; Blair et al., 2006; Xiao-Bin et al., 2006; Xiao-Gang et al., 2006; Schnitzer et al., 2006; Holland, 2004; entre otros). Estos trabajos confirman las ventajas agronómicas y medioambientales de la agricultura de conservación. Sin embargo, no se llevó a cabo ningún estudio detallado en el ámbito europeo y, por eso, algunos de los descubrimientos han de ser utilizados con precaución hasta que se verifiquen, a gran escala, en distintos tipos de climas, cultivos y tipos de suelos (Holland, 2004).

En Portugal, ya se han realizado algunos estudios sobre este problema bajo distintas perspectivas, de los cuales se pueden citar los trabajos, entre otros, de: Carvalho (2002); Basch y Carvalho (1997a); Basch y Carvalho (1997b); Basch y Carvalho (1996). En estos trabajos, ya se ha demostrado que con la práctica de la siembra directa en cultivos de cereales de secano, en climas mediterráneos, se alcanzan producciones semejantes a las obtenidas con el sistema de laboreo tradicional. De este modo, la tecnología utilizada en la producción, así como sus consecuencias en el suelo, asumen un papel importante en la conservación del suelo sin perjudicar la productividad.

Por todo lo anterior, el objetivo de este trabajo es evaluar los beneficios potenciales de un sistema de conservación de cereales de secano, en un suelo de textura muy fina, con clima mediterráneo, en un país europeo. Estos beneficios son evaluados a través del análisis de la alteración del comportamiento y de la correlación entre diversas características de los suelos, cuando se altera el sistema de producción convencional o tradicional (ST) para un sistema de conservación que recurre a la siembra directa durante 10 años consecutivos (SD). 


\section{MATERIAL Y MÉTODOS}

El estudio tuvo lugar en un ensayo de producción de cereales de secano, ubicado en una unidad homogénea de terreno, con un declive del $2 \%$, en el sur de Portugal ( $37^{\circ} 59^{\prime} \mathrm{N}$ : $7^{\circ} 57^{\prime} \mathrm{W}$ ), con clima mediterráneo, en un suelo tipo Vertic Cambisol, de acuerdo con la World Reference Base for Soil Resources WRBSR (IUSS Working Group WRB, 2006), típico de las condiciones edafoclimáticas mediterráneas.

La nomenclatura de horizontes y capas de suelo utilizada en el trabajo aquí presentado están de acuerdo con lo indicado en Soil Survey Staff (SSS, 1981). En esta unidad homogénea de Vertic Cambisol, está instalada una rotación de cultivos tradicionales de secano, típica de la región (Girasol Trigo Mole - Cebada Dística).

En una sub-unidad de terreno los cultivos están sometidos, desde hace más de 50 años, al sistema de producción tradicional, con ayuda de las técnicas de movilización de suelo tradicionales (ST). En otra sub-unidad, se respetó los mismos cultivos, bajo condiciones de un sistema de producción de conservación que en los últimos 10 años consecutivos, recibió un conjunto de prácticas agrícolas que utilizaban las siembras directas (SD10).

En el sistema tradicional o convencional (ST) se llevaron a cabo las siguientes prácticas agrícolas: labranzas de $30 \mathrm{~cm}$ de profundidad para el control de plantas espontáneas; labradas a $30 \mathrm{~cm}$ de profundidad y gradeos cruzados para preparar la capa de la semillas; siembras; rastrillajes; mondaderas mecánicas; tratamientos fitosanitarios y abonos. En el sistema de conservación con siembra directa (SD10) se llevaron a cabo las siguientes prácticas agrícolas: control de plantas espontáneas en la pre-siembra con un herbicida total sistémico no residual; siembra directa y abonos.

En cada uno de los sistemas de producción, se seleccionaron, al azar, 3 bloques (repeticiones), donde se abrieron perfiles de observación y recolección de muestras, en un total de 6 perfiles. Con objeto de diferenciar los efectos del trabajo en el suelo a distintas profundidades y sus relaciones con los horizontes de cada perfil, en cada bloque, se recogieron cinco muestras o repeticiones con cerca de un kilo de material terroso cada y cinco anillos de muestras no perturbadas. Cada cinco muestras han sido retiradas de 10 en $10 \mathrm{~cm}$ de profundidad hasta las capas C (SSS, 1981).

Con el fin de evaluar las características consideradas como indicadoras de las consecuencias de las alteraciones en el sistema de producción, cada muestra fue sometida a diversos análisis en laboratorio con tres lecturas por cada una. Las recolecciones de muestras y respectivos análisis fueron siendo efectuadas al largo de los 10 años consecutivos.

Las características o propiedades evaluadas fueron las siguientes:

- Capacidad de Campo (pF 2,54), a través de anillos con muestras no perturbadas, en placas de presión (Veihmeyer y Hendrickson, 1949);

- Coeficiente de Marchitamiento (pF 4,2), utilizando el método de las membranas de presión (Veihmeyer y Hendrickson, 1949);

- contenido en Materia Orgánica (MO), determinado por el método de destrucción de la misma a través de la oxidación por vía húmeda (Walkley-Black, 1934);

- Nitrógeno Total (N), por el método Kjeldahl (Santos, 1991);

- razón Carbono/ Nitrógeno $(\mathrm{C} / \mathrm{N})$, calculada matemáticamente con los dados del contenido en $\mathrm{MO}$ y N (Nelson y Sommers, 1982);

- Cantidad, Dimensiones de los agregados y Coeficiente de Agregación por el método de la criba húmeda (Yoder, 1936);

- Densidad Aparente (DAP), por el método de los anillos con muestras no perturbadas (Burke et al., 1986).

Se realizaron 15 evaluaciones con tres lecturas por cada una, para cada característica y cada $10 \mathrm{~cm}$ de profundidad, hasta los $40 \mathrm{~cm}$, en cada sistema de producción, con un total de 120 análisis y 360 lecturas por indicador. 
También se realizó, la descripción de la macroestructura de campo (tipo, clase y grado de los agregados), según la FAO/UNESCO (1976).

Los datos obtenidos para los parámetros estudiados fueron procesados en el paquete estadístico SPSS (SPSS, 2007). Se analizaron y se compararon, a través de los valores de las medias de cada conjunto de quince lecturas, para cada indicador y del análisis de la varianza y del coeficiente de variación. Se evaluaron, igualmente, las correlaciones de Pearson ( $r)$, y su respectiva significancia $(p)$ al nivel del 5\%, para analizar las relaciones de cada indicador, a cada profundidad, entre los diversos perfiles.

\section{RESULTADOS Y DISCUSIÓN}

Los resultados de los análisis de cada indicador en cada profundidad a lo largo de los 10 años consecutivos en el sistema tradicional (ST), obtuvieron un coeficiente de variación muy pequeño (2,9 $\%$ ) entre el primero y el $10^{\circ}$ año en estudio. Así, se consideró que la media de los 10 años consecutivos es una buena representación de cada característica en cada profundidad del ST.

En el caso del sistema de siembras directas (SD10), los resultados de los análisis de cada indicador en cada profundidad, se fueron modificando muy ligera y progresivamente a lo largo de los 10 años consecutivos. Dado que esas alteraciones se dieron siempre en el mismo sentido, se consideró innecesario incluir el presente trabajo todos los resultados durante este período. Así, por que se consideran suficientes para comparar la diferencia de sistemas al fin de los referidos años, para SD10, se presentan aquí sólo los resultados obtenidos para cada característica en cada profundidad del $10^{\circ}$ año de ensayo.

En los gráficos de las Figuras 1 y 2 se pueden observar los resultados obtenidos con las evaluaciones echas para cada una de las características en estudio. En ellos se aprecia, no sólo la variación de los distintos indicadores entre los dos perfiles correspondientes a los sistemas estudiados (SD10 y ST), sino también el comportamiento de cada uno de ellos en profundidad, e incluso la propia relación con la diferencia de capas presentada en los perfiles.

Las correlaciones de Pearson ( $r$ ), y la significancia ( $p$ ), de cada indicador, a cada profundidad, entre los distintos perfiles $(n=3)$ de cada sistema de producción, resultaron positivas variando entre valores de $r=0,811$ y 0,879 y valores de $p>0,07$ y 0,09. Para los mismos indicadores a diferentes profundidades entre perfiles de sistemas diversos, la correlación no resultó significativa, variando entre $p<0,01$ y 0,02 . Estos valores, indican que los promedios de las 5 repeticiones por cada tres perfiles, son representativos del comportamiento de cada indicador en cada profundidad y para cada sistema de producción. De este modo, los diversos resultados se presentan, en las figuras, en relación a un perfil-tipo para cada sistema de producción, los cuales se constituyen por el promedio de cada variable para cada profundidad. Los valores de $(r)$ y $(p)$ confirman, igualmente, la hipótesis de que las diferencias encontradas en cada indicador, a cada profundidad, están vinculadas a la diferencia en el sistema de producción. Estos resultados demuestran que, tanto la Capacidad de Agua Utilizable (CU), como el contenido en Materia Orgánica (MO) y la razón $\mathrm{C} / \mathrm{N}$, así como el Coeficiente de Agregación (la estabilidad y cantidad de los agregados), aumentaron en el sistema de siembra directa, mientras que la Densidad Aparente, disminuyó. Estas diferencias resultan más notorias en las capas por debajo de los $20 \mathrm{~cm}$ de profundidad.

Se debe tomar en cuenta que a partir del análisis de estas figuras se puede deducir que:

El contenido de (MO), excede el $2 \%$ en la superficie del sistema SD10, consiguiendo mantenerse, hasta los $30 \mathrm{~cm}$ de profundidad en valores muy semejantes a los presentados en la capa superficial del sistema ST. Se considera muy bueno que, en cereales de secano en un clima mediterráneo, se alcance un contenido por encima del $2 \%$ de (MO) lo cual se refleja en muchas otras características interrelacionadas con él. De hecho, la erosión ocurre cuando el contenido de (MO), del suelo cae por debajo de 2\% (Greenland et al., 1975). Este contenido se debe, no solo a la cantidad de residuos orgánicos dejados en el suelo por este sistema, sino también, al hecho de que estos residuos sufren mayor humificación que en el sistema tradicional. Además, se puede analizar la humificación y la 


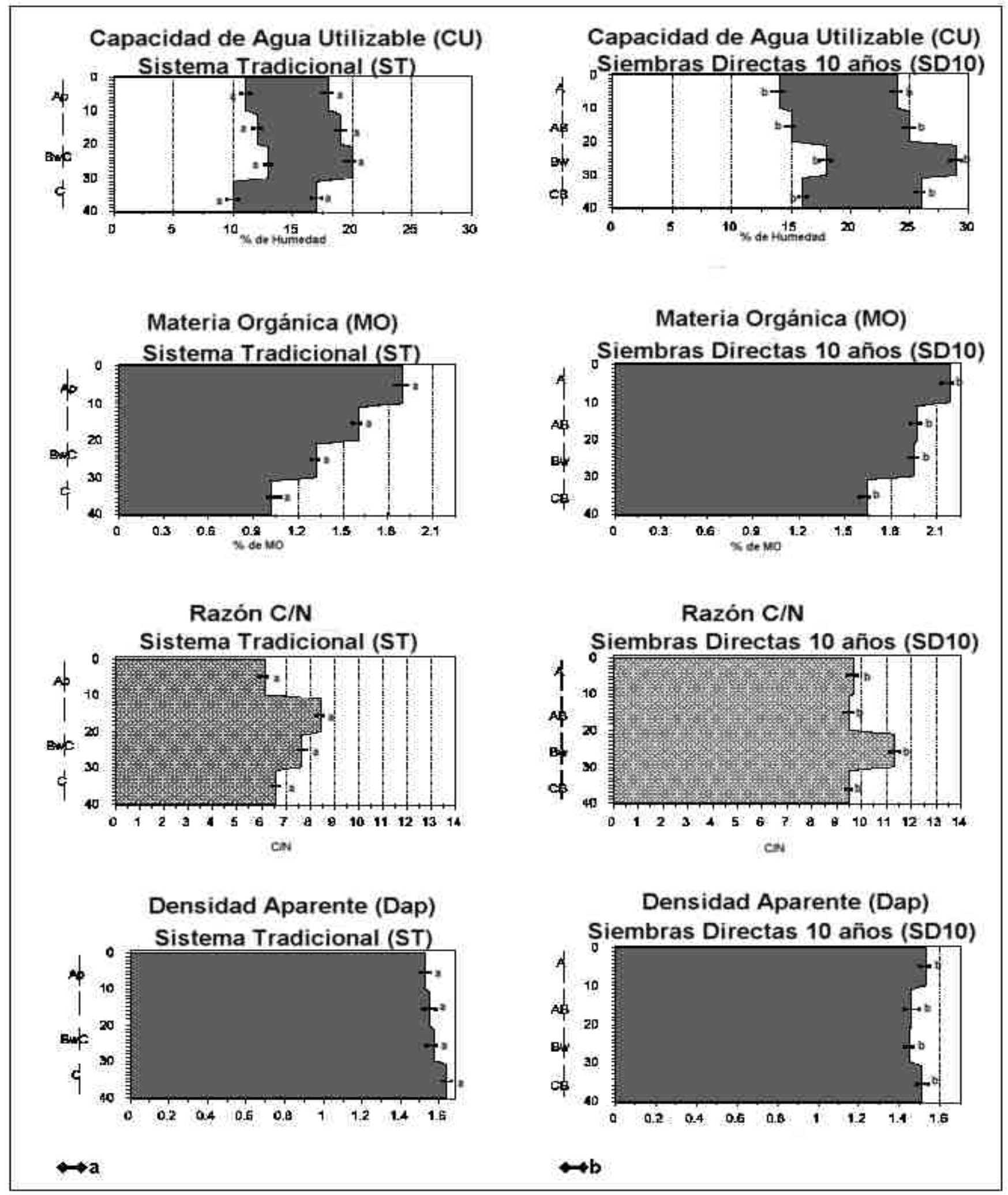

Fig. 1: Gráficos de comparación entre ST/SD10

[letras distintas $(a: b)$ indican diferencias significativas entre sistemas $(p<0,02)$ ] 


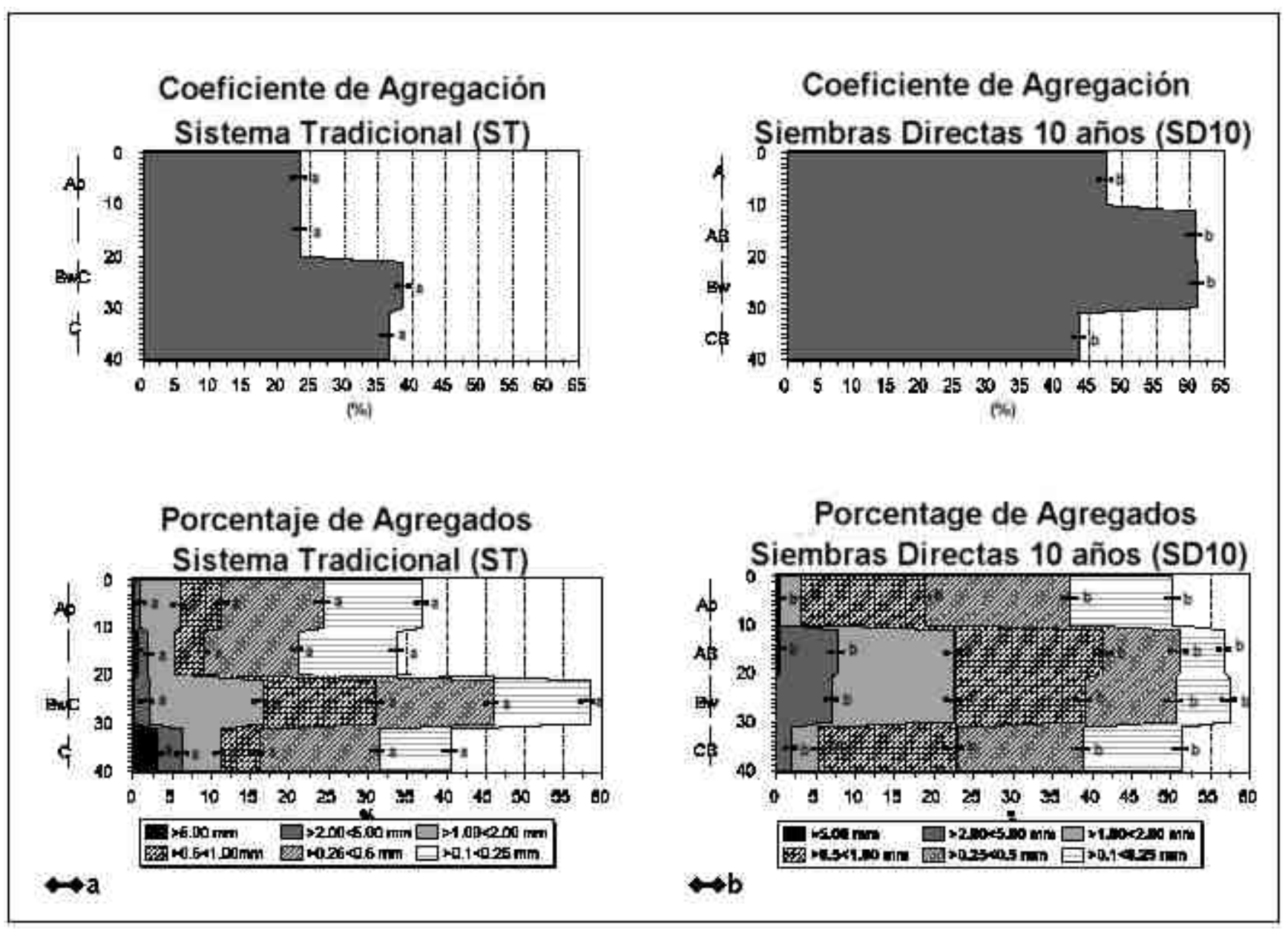

Fig. 2: Continuación de gráficos de comparación entre ST/SD10

[letras distintas $(a: b)$ indican diferencias significativas entre sistemas $(p<0,02)$ ]

mineralización con los resultados de la razón $\mathrm{C} / \mathrm{N}$, los cuales, además de ser notoriamente superiores en el sistema SD10, disminuyen poco con la profundidad, lo que demuestra la capacidad de este sistema de conservación para recuperar las competencias de humificación de los residuos orgánicos dejados en el suelo. Esto viene a confirmar lo que Xiao-Gang et al. (2006), han concluido al verificar que el contenido en arcilla de los suelos ejerce una protección contra la mineralización y contribuye para la humificación.

Dado que la humificación aumenta en el sistema SD10 a pesar de mantenerse alguna mineralización, no excesiva, resulta una actividad microbiana importante para el abastecimiento de nitrógeno de los cultivos, y la simultánea reducción de $\mathrm{CO}_{2}$ en la atmósfera causada por el secuestro en el suelo. Además, el contenido de materia orgánica en SD10, es superior incluso en profundidad, por tanto, su influencia benéfica en el suelo, alcanza capas mas alejadas de la superficie, lo que confirma, una importante capacidad para contribuir en el secuestro de $\mathrm{CO}_{2}$. Así, se confirma, para estas condiciones edafoclimáticas, lo que muchos autores ya han concluido, para otras condiciones, entre ellos Bayer et al. (2001), que han afirmado que la estabilidad física de la materia orgánica es muy importante en la conservación y en el aumento de la calidad del suelo y, determina el potencial del suelo para actuar como un transformador de $\mathrm{CO}_{2}$ atmosférico. De la misma forma, Xiao-Gang et al. (2006), afirmaron que el contenido en MO es de vital importancia para evaluar la sustentabilidad de las prácticas culturales, en suelos desérticos.

En lo que respecta a la Densidad Aparente, ésta muestra valores muy semejantes en las capas superficiales de ambos sistemas, a pesar de la ausencia de movilización en el sistema SD10. Sin embargo, por debajo de los $10 \mathrm{~cm}$ de profundidad, en el sistema tradicional, se comprueba un aumento progresivo en profundidad. En este sistema, las labranzas del suelo crean una porosidad 
artificial en las capas superficiales, provocando una aparente descompactación pero, en realidad, la estructura de estos horizontes se degrada. La estabilidad, la cantidad y la dimensión de los agregados de estas capas sufren alteraciones y los horizontes sub-superficiales sufren las consecuencias del exceso de paso de maquinaria sobre un suelo con una capa Bw (SSS, 1981) que inevitablemente, se compacta. Además de eso, tal como afirman Riley et al. (2008), cuando el aumento de la DAP es acompañado por un aumento de la MO es de esperar que aumente el número de agregados de pequeña dimensión y su estabilidad con aumento de la capacidad de agua utilizable para las plantas (CU).

En el sistema de conservación, aumentó significativamente el Coeficiente de Agregación no sólo, sino que también aumentó el porcentaje total de agregados, especialmente los que pertenecen a las clases de menor dimensión. Además se comprobó una estabilidad de los resultados en profundidad en todo el perfil. También se observó que en el sistema de conservación, los agregados de mayor dimensión están presentes en mayor cantidad y en todo el perfil, mientras que en el sistema tradicional, estos aparecen sobre todo debajo de la profundidad de las labradas, lo que es bastante ilustrador de la influencia perturbadora de las mismas, en el equilibrio natural de las características del suelo. Resultados semejantes fueron obtenidos por Xiao-Gang et al. (2006) en suelos desérticos a lo que observen una correlación positiva entre la estabilidad de los agregados y el contenido en MO.

Tabla 1: Descripción de la estructura de campo según FAO/UNESCO (1976)

\begin{tabular}{|c|c|c|}
\hline ST & & SD10 \\
\hline Primavera & & Primavera \\
\hline 5 & $\begin{array}{l}\text { PROFUNDIDAD } \\
\text { (cm) }\end{array}$ & 5 \\
\hline Ap (SSS, 1981) & CAPA & A (SSS, 1981) \\
\hline Anisoforme Angulosa & TIPO & $\begin{array}{l}\text { Granulosa + } \\
\text { Anisoforme Angulosa a Sub-angulosa }\end{array}$ \\
\hline Fina a Media & CLASE & Media a Fina \\
\hline Fuerte a Muy Fuerte & GRADO & Fuerte \\
\hline 23 & $\begin{array}{l}\text { PROFUNDIDAD } \\
(\mathrm{cm})\end{array}$ & 15 \\
\hline BWC (SSS, 1981) & CAPA & $A B(S S S, 1981)$ \\
\hline $\begin{array}{l}\text { Prismática }+ \\
\text { Anisoforme Angulosa a Sub-angulosa }\end{array}$ & TIPO & $\begin{array}{c}\text { Prismática + } \\
\text { Anisoforme Angulosa a Sub-angulosa }\end{array}$ \\
\hline Media & CLASE & Media a Grande \\
\hline Fuerte a Muy Fuerte & GRADO & Fuerte \\
\hline & $\begin{array}{l}\text { PROFUNDIDAD } \\
(\mathrm{cm})\end{array}$ & 25 \\
\hline & CAPA & Bw (SSS, 1981) \\
\hline & TIPO & $\begin{array}{c}\text { Prismática }+ \\
\text { Anisoforme Sub-angulosa }\end{array}$ \\
\hline & CLASE & Media a Grande \\
\hline & GRADO & Fuerte a Muy Fuerte \\
\hline & $\begin{array}{l}\text { PROFUNDIDAD } \\
(\mathrm{cm})\end{array}$ & 35 \\
\hline & CAPA & CB (SSS,1981) \\
\hline & TIPO & Anisoforme Angulosa \\
\hline & CLASE & Media a Grande \\
\hline & GRADO & Fuerte a Muy Fuerte \\
\hline
\end{tabular}

Uniendo estos resultados con la descripción de la macro estructura de campo, que se puede observar en la Tabla 1, se puede comprobar que el sistema de conservación, en relación con el 
sistema tradicional, favorece, en la capa superficial, clases de agregados semejantes, con un grado un poco más ligero y de tipo mixto con granuloso. En los horizontes por debajo de los $20 \mathrm{~cm}$ de profundidad, el sistema SD10, en relación al ST, favorece la formación de agregados de clase más gruesa, al tiempo que mantiene las demás características. Estos resultados son complementarios a todos los anteriores, en el sentido de que resaltan la recuperación promovida por la no movilización del suelo, en lo que respecta a sus características intrínsecas. De esta forma, se confirma una vez más, que en estas condiciones edafoclimáticas, se verifica lo mismo que otros autores ya concluyeron para otras condiciones. Por ejemplo, Schwab et al. (2002) demostraron que de la movilización convencional se obtiene una mayor compactación del suelo, en comparación con otros tratamientos de suelo;

La Capacidad de Almacenamiento de Agua del suelo y la Capacidad de Agua Utilizable aumentaron de forma considerable en el sistema de conservación a lo largo de todo el perfil. Este hecho representa un gran salto cualitativo en la capacidad de los suelos para resistir a la desertificación, la cual no es más que una consecuencia de la incapacidad del suelo para retener agua y, por tanto, conservar la vida vegetal, base funcional de las cadenas alimenticias. Estos resultados son muy semejantes a los que Govaerts et al. (2006) obtuvieron en México bajo condiciones semiáridas en un estudio con siembras directas en una cultura de maíz de sequero. Según estos autores, la siembra directa conjugada con rotaciones donde los residuos son dejados en el suelo, aumenta la disponibilidad de agua para las plantas, mejora la estructura, los nutrientes disponibles y la biodiversidad microbiológica.

En el sistema SD10, el perfil del suelo, pierde las características típicas de la degradación resultante de la influencia humana sobre la capa superficial, al mismo tiempo que logra una mayor diferenciación de horizontes en profundidad, propiciando la formación de un horizonte Bw (SSS, 1981) bien definido. Por otra parte, a $40 \mathrm{~cm}$ de profundidad, el perfil del sistema SD10 aún presenta una capa de transición, BC (SSS, 1981) con mayor potencialidad para la exploración de las raíces de los cultivos que el perfil del sistema ST, que presenta un horizonte BwC (SSS, 1981) a partir de los $20 \mathrm{~cm}$ de profundidad y un C (SSS, 1981) a partir de $10 \mathrm{~cm} 30 \mathrm{~cm}$.

\section{CONCLUSIONES}

Después del análisis y discusión de los resultados presentados, puede concluirse que en regiones interiores de Climas Mediterráneos en Europa, en un "Vertic Cambisol":

- El sistema de agricultura de conservación, que recurre a la siembra directa durante períodos prolongados de forma consecutiva, produce alteraciones benéficas en el contenido de Materia Orgánica, en la razón $\mathrm{C} / \mathrm{N}$, en la Densidad Aparente, en la Estabilidad de Agregación. Así la agricultura puede tener un papel favorable para en cuanto a la capacidad del suelo para producir vegetales, gestionar el régimen hídrico, secuestrar carbono orgánico y resistir a la erosión;

- Todas las características mejoradas por el sistema de conservación, permitieron que el suelo presentara una mayor diferenciación de capas y un mayor espesor del perfil, mayor estabilidad en sus características, menor compactación, mayor capacidad de retención de agua y por lo tanto, mejor equilibrio entre aireación y humedad, alcanzando mejores condiciones para el desarrollo de hábitat funcionales y para la producción vegetal, fuente de energía indispensable para la vida;

- El sistema de producción que recurre a la siembra directa, demostró tener, no solo un potencial de conservación, sino también, un potencial de recuperación de este recurso. Esto representa una importante alternativa cuanto a la reducción de procesos graves de degradación medioambiental inducidos por la agricultura.

- La siembra directa puede ayudar en la solución de problemas de reconversión energética y, aporta respuestas inequívocas sobre el papel benéfico de los sistemas de producción agrícola de conservación en la sociedad y en la naturaleza del futuro. 
- Las opciones relacionadas con sistemas de producción conservadores como el de la siembra directa que deja residuos de cultura en el suelo y excluye las movilizaciones, permiten hacer agricultura sustentable en suelos de textura fina de regiones mediterráneas continentales de Europa.

\section{REFERENCIAS}

Abrisqueta J.M. y otros cuatro autores; Effects of soil tillage on runoff generation in a Mediterranean Apricot Orchard, Agricultural Water Management: 93(1-2), 11-18 (2007).

Basch G. y M. Carvalho; No-tillage crop production for erosion prevention, Proceedings of the International Conference on Mediterranean Desertification: 2, 431-441, Greece 29 Octubre a 1 Noviembre (1996).

Basch G. y M. Carvalho; Perspectives of conservation tillage in Portugal, Actas do Congresso Nacional sobre Agricultura de Conservacion: Rentabilidad y Medio Ambiente: 3, 21-37, Córdoba 2 a 4 de Octubre(1997a).

Basch G. y M. Carvalho; Economical considerations on no-tillage crop production in Portugal, Proceedings of the EC - Workshop on Experience with the applicability of no-tillage crop production in the West-European countries: 4, 17-24, Boigneville, France 24 a 26 de Junio (1997b).

Bastin G.N. y otros cuatro autores; Indicators of landscape function: comparing patchiness metrics using remotely-sensed data from rangelands, Ecological Indicators: 1(4), 247-260 (2002).

Bayer C. y otros cuatro autores; Changes in soil organic matter fractions under subtropical no-till cropping systems, Soil Science Society of America Journal: 65, 1473-1478 (2001).

Blair N., R.D. Faulkner, A.R. Till y P.R. Poulton; Long-term management impacts on soil C, N and physical fertility - Part I: Broadbalk experiment, Soil and Tillage Research: 91(1), 30-38 (2006).

Burke W., D. Gabriels y J. Bouma; Soil Structure Assessment, 24-26, A. A. Balkema Publishers, Rotterdam/Boston (1986);

Castro J. y otros cinco autores; Effects of different olive-grove management systems on organic carbon and nitrogen content of the soil in Jaén (Spain), Soil and Tillage Research: 98(1), 56-67 (2008).

Carvalho M.; Sementeira directa - Aspectos agronómicos e edáficos, Actas do $1^{\circ}$ Congresso Nacional de Mobilização de conservação do solo: 39-50, Évora, Portugal 12 a 14 de Junio (2002).

Carvalho M. y otros cuatro autores; A sementeira directa e os resíduos das culturas no aumento do teor de matéria orgânica do solo e na resposta da cultura do trigo à adubação azotada, Actas do $1^{\circ}$ Congresso Nacional de Mobilização de Conservação do Solo: 185-191, Évora, Portugal 12 a 14 de Junio (2002).

Chatskikh D. y J.E. Olesen; Soil tillage enhanced $\mathrm{CO}_{2}$ and $\mathrm{N}_{2} \mathrm{O}$ emissions from loamy sand soil under spring barley, Soil and Tillage Research: 97(1), 5-18 (2007).

Elder J.W. y L. Rattan; Tillage effects on gaseous emissions from an intensively farmed organic soil in North Central Ohio, Soil and Tillage Research: 98(1), 45-55 (2008).

FAO-UNESCO; Adiestramiento para el desarrollo agropecuario y rural, OIT, Roma, Italia (1976).

Govaerts B. y otros cinco autores; Long-term consequences of tillage, residue management, and crop rotation on maize/Wheat root rot and nematode populations in subtropical highlands, Applied Soil Ecology: 32(3), 305-315 (2006). 
Govaerts B. y otros siete autores; Influence of tillage, residue management, and crop rotation on soil microbial biomass and catabolic diversity, Apllied Soil Ecology: 37(1-2), 18-30 (2007).

Greenland D.J., D. Rimmer y D. Payne; Determination of the structural stability class of English and welsh soils, using a water-coherence test, Journal of Soil Science: 26, 303- 311 (1975).

Hermle S., T Anken, J. Leifeld y P. Weisskopf; The effect of the tillage system on soil organic carbon content under moist, cold-temperate conditions, Soil and Tillage Research: 98(1), 94-105 (2008).

Holland J.M.; The environmental consequences of adopting conservation tillage in Europe: reviewing the evidence, Agriculture, Ecosystems and Environment: 103, 1-25 (2004).

IUSS Working Group WRB; World Reference Base for Soil Resources 2006, $2^{\text {nd }}$ edition, World Soil Resources Reports: 103, FAO, Rome (2006);

Jin K. y otros diez autores; Effects of different management practices on the soil-water balance and crop yield for improved dryland farming in the Chinese Loess Plateau, Soil and Tillage Research: 96(1-2), 131-144 (2007).

Lal R., D.C. Reicosky y J.D. Hanson; Evolution of the plow over 10,000 years and the rationale for notill farming, Soil and Tillage Research: 93(1), 1-12 (2007).

Moreno R.G. y otros cuatro autores; Tillage and soil type effects on soil surface roughness at semiarid climatic conditions, Soil and Tillage Research: 98(1), 35-44 (2008).

Nelson D.W. y L.E. Sommers; Total carbon, organic carbon, and organic matter, In Methods of Soil Analysis, Part 2, Chemical and Microbiological Properties by A. L. Page et al., pp 539-579 American Society of Agronomy, Madison (1992)

Pereira J. O., P. Défossez y G. Richard; Soil susceptibility to compaction by wheeling as a function of some properties of a silty soil as affected by the tillage system, European Journal of Soil Science: 58 , 34-44 (2007).

Reynolds W. D. y otros cinco autores; Land management effects on the near-surface physical quality of a clay loam soil, Soil and Tillage Research: 96(1-2), 316-330 (2007).

Riley H. y otros cuatro autores; Soil structure, organic matter and earthworm activity in a comparison of cropping systems with contrasting tillage, rotations, fertilizer levels and manure use, Agricultural, Ecosystems \& Environment: 124(3-4), 275-284 (2008)

Santos J.Q.; Fertilização - Fundamentos da utilização dos adubos e correctivos, Publicações Europa - América, Lisboa, Portugal (1991).

Schnitzer M. y otros cuatro autores; Long-term cultivation effects on the quantity and quality of organic matter in selected Canadian prairie soils, Geoderma: 130 (1-2), 141-156 (2006).

Schwab E.B., D.W. Reeves, C.H. Burmester y R.L. Raper; Conservation tillage systems for cotton in the Tennessee Valley, Soil Science Society of America Journal: 66 (2), 569-577 (2002).

SPSS; Statistical Package for Social Sciences for Windows, Version 16. SPSS Inc. Chicago, Illinois, USA (2007)

SSS, Soil Survey Staff; Soil Survey Manual, United States Department of Agriculture. Soil Conservation Service, Directive 430-V, Washington, USA (1981)

Veihmeyer F.J. y A.H. Hendrickson; Methods of measuring field capacity and permanent wilting percentage of soils, Soil Science: 68, 75-94 (1949) 
Walkley A. y I.A. Black; An examination of Degtjareff method for determining soil organic matter and a proposed modification of the chromic acid titration method, Soil Science: 37, 29-38 (1934);

Xiao-Bin W. y otros cuatro autores; Potential effect of conservation tillage on sustainable land use: a long review of global long-term studies, Pedosphere: 16(5), 587-595 (2006).

Xiao-Gang L. y otros cuatro autores; Cultivation effects on temporal changes of organic carbon and aggregate stability in desert soils of Hexi Corridor region in China, Soil and Tillage Research: 91 (1-2), $22-29$ (2006).

Xiao-Gang L. y otros cuatro autores; Soil management changes organic carbon pools in alpine pastureland soils, Soil and Tillage Research: 3(1), 186-196 (2007).

Yoder R.E.; A direct method of aggregate analysis of soils and a study of the physical nature of erosion losses, Journal of American Society Agronomics: 28, 337-351 (1936) 
